4th International Conference Photoinduced Phase Transitions and Cooperative Phenomena, Wrocław 2011

\title{
White Light Snapshot of Non-Equilibrium High-Spin State in a Molecular Crystal
}

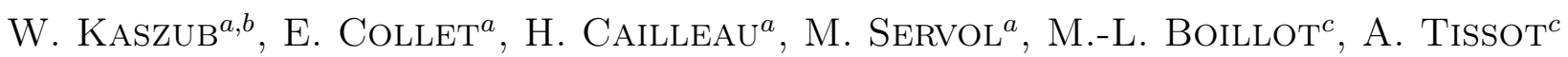 \\ AND M. LORENC ${ }^{a, *}$ \\ ${ }^{a}$ Institut de Physique de Rennes, UMR CNRS 6026, Université de Rennes 1, Campus Beaulieu \\ 35042 Rennes, France \\ ${ }^{b}$ Quantum Electronics Laboratory, Faculty of Physics, Adam Mickiewicz University, 60-614 Poznań, Poland \\ ${ }^{c}$ Institut de Chimie Moleculaire et Matériaux d'Orsay, UMR CNRS 8182, Université Paris-Sud 11 \\ 91405 Orsay, France
}

\begin{abstract}
The spin-crossover compound [(TPA) $\mathrm{Fe}(\mathrm{TCC})] \mathrm{PF}_{6}$ was experimentally investigated using time-resolved optical pump and supercontinuum probe technique. Changes of VIS optical density at thermal equilibrium of a crystal with different high-spin/low-spin fractions are compared to changes recorded picoseconds after femtosecond laser excitation. A white light snapshot of the high-spin state at $180 \mathrm{~K}$ is extracted from time-resolved spectra. Our results indicate that the temperature of the electronic state affects the absorption spectra of high- and low-spin states.
\end{abstract}

PACS: 82.53.Xa, 78.47.D-, 42.70.Gi, 31.70.Ks

\section{Introduction}

$[(\mathrm{TPA}) \mathrm{Fe}(\mathrm{III})(\mathrm{TCC})] \mathrm{PF}_{6}$ belongs to the family of spin crossover compounds, which are prototypes of molecular bistability in solid state. In such compounds, molecules interconvert between two different stable states corresponding to the low-spin and high-spin states (LS and HS, respectively). Switching between these two states occurs under external stimuli such as temperature, pressure, or light $[1,2]$. Changes in fraction of HS and LS molecules constituting the crystal can be detected using variety of techniques, including magnetic measurements, optical transmission or reflectance measurements, X-ray diffraction and absorption measurements. Spin changes induced by light hold big promise for application purposes, and numerous studies prove the point. Beside continuous wave $(\mathrm{cw})$ and nanosecond lasers, the femtosecond laser pulses are a formidable gateway to the control over materials. They allow a macroscopic number of molecules be excited on the time scale of electronic transitions, thus triggering coherently a sequence of processes starting from the molecule and ending on the macroscopic entity - the crystal.

Direct transitions from LS to HS quantum states are forbidden. To bypass this limitation, a higher lying ligand to metal charge transfer (LMCT) state is directly populated from the LS ground state, so that its relaxation opens pathway to metastable HS state. Energy of a single laser photon can be 10-100 times higher than the energy difference between spin states being inter-switched. The excess energy imparted by the photon on

* corresponding author; e-mail: maciej.lorenc@univ-rennes1.fr the molecule is dissipated through thermal excitations of the molecular and lattice vibrational modes. First experimental evidences of HS state generation with a femtosecond pulse were recently reported for the compound $[(\mathrm{TPA}) \mathrm{Fe}(\mathrm{TCC})] \mathrm{PF}_{6}[3,4]$. In this last study, the time course of the optical transmission on two specific wavelengths, one on either side of the isosbestic point, revealed the photogeneration of high-spin species at $T_{1 / 2}$ within 300 fs following excitation. Little though could be inferred on the spectral behaviour of the transient species. In this report we attempt extracting the spectrum of the photogenerated state of the crystal immediately after relaxation from the LMCT states.

\section{Experimental}

The experiment was performed on the monoclinic polymorph [5] of [(TPA)Fe(TCC)] $\mathrm{PF}_{6}$. The monocrystals used had plate shape and were selected for size, typically $200 \times 50 \mu \mathrm{m}^{2}$. Femtosecond oscillator (Mira, Coherent) followed by the regenerative amplifier (Legend Elite II) generates a train of $100 \mathrm{fs}$ long laser pulses at $800 \mathrm{~nm}$ and $1 \mathrm{kHz}$ repetition rate. This frequency is slowed down to $500 \mathrm{~Hz}$ by a mechanical chopper phase locked to the laser. After the chopper the beam is split by a $60 / 40$ beamsplitter. The more intense beam, the pump, is optically delayed with respect to the probe, then chopped once more down to $250 \mathrm{~Hz}$. The less intense beam is focused on a sapphire window to generate white light supercontinuum. These pulses are chirped and having acquired additional chromatic dispersion in the sapphire window, their group velocity dispersion is equal to $10 \mathrm{fs} / \mathrm{THz}$. The white light pulses are further split in two parts: one serves as probe and the other as reference in the experiment. Finally, pump and probe 
are spatially overlaid on the crystal surface with the aid of a microscope objective (InFocus, model KC, Infinity, $\mathrm{NA}=0.05)$. Both pulses are polarized parallel to the long crystal axis.

The white light spectra were dispersed with a spectrometer (Acton Reserach Corporation - SPECTRAPro 2500i) and imaged on a CCD detector (Princeton Instruments PIXIS 100). For every time delay, 10000 spectra were collected, averaged and analysed using double reference method to improve the signal to noise ratio [6]. Care was taken to avoid outliers in averaging and to ensure the correct sign of the optical density change. In brief, without pump induced effect, by using double reference method the signal equals one, and the scatter around this value results from statistical noise. In the presence of pump induced effect, the signal deviates from one and the amplitude of this deviation should be directly proportional to the quantum efficiency of the photoinduced process. In stationary experiments, where no pump beam was incident on the sample, the signal was simply divided by the reference thereby recording changes in optical density as a function of temperature. The ratio between signal and reference was checked before each experiment.

\section{Results and discussion}

\subsection{Spectral kinetics at equilibrium}

In the studied compound, the fraction of HS molecules changes gradually with temperature. It is equal to 0.5 at the transition temperature $T_{1 / 2}=214 \mathrm{~K}$. This gradual evolution is characteristic of a weakly cooperative material, as opposed to strongly cooperative systems undergoing a first-order phase transition, manifested by an abrupt change.

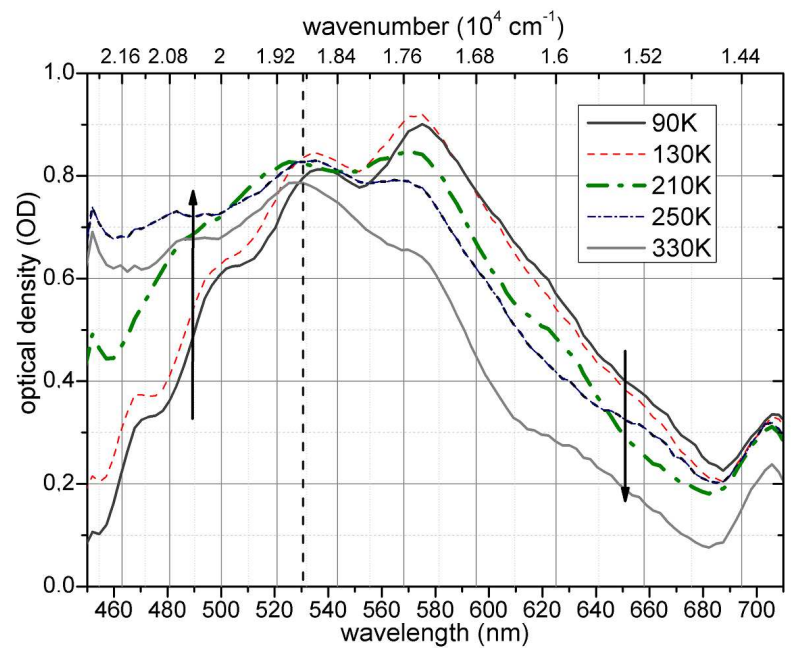

Fig. 1. Temperature dependence of the optical absorption of $[(\mathrm{TPA}) \mathrm{Fe}(\mathrm{TCC})] \mathrm{PF}_{6}$.

Figure 1 shows VIS spectra taken at different temperatures, starting at $90 \mathrm{~K}$ up to $330 \mathrm{~K}$, the temperatures at which the fraction of HS forms varies between 0 and 0.9 , respectively. The observed gradual change of the optical density is reminiscent of the spin-crossover evolution. These absorption bands are typical for catecholato-to iron(III) charge transfer transitions. Below $530 \mathrm{~nm}$, the optical density increases as the crystal converts from LS to HS states. At wavelengths above $530 \mathrm{~nm}$, OD exhibits the opposite behaviour.

Another interesting feature that can only be observed using broad bandwidth spectroscopy is the vibrational contribution riding on the electronic spectrum and clearly prevailing at low temperatures. The interval between these apparent sub-bands of ca. $1270-1310 \mathrm{~cm}^{-1}$, can be tentatively attributed to a vibrational mode strongly coupled to the optical LMCT transition, very likely the CO stretching mode of the TCC ring at $1274 \mathrm{~cm}^{-1}[7,8]$. The vibrational contribution is gradually smeared out by rising temperature, and becomes hardly distinguishable at $330 \mathrm{~K}$. In order to understand the spectral kinetics during spin crossover we tried a simple model in which we assume that the spectrum at any temperature is a sum of LS and HS spectra weighted by their respective molecular fractions. HS fraction can be extracted from temperature dependent magnetic measurements [5]. The LS spectrum is directly obtained at $90 \mathrm{~K}$, whereas the HS spectrum is obtained from measurements at $330 \mathrm{~K}$ after correcting for a 0.1 contribution from LS state.

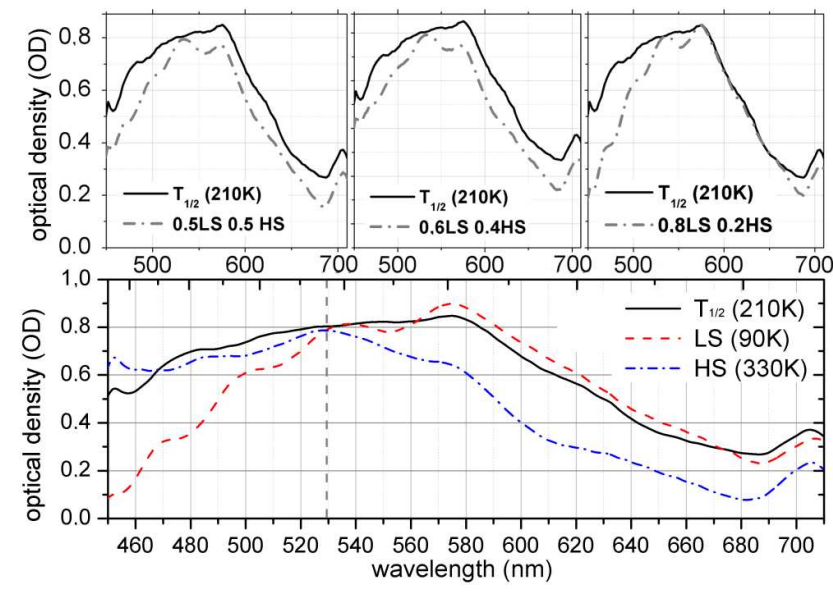

Fig. 2. Lower part: spectra of LS, HS and crossover state at $210 \mathrm{~K}$, upper part: spectral reconstruction of optical density at $210 \mathrm{~K}$ based on weighing two contributions: LS and HS state spectra.

Figure 2 shows the LS and HS spectra together with the spectrum at $T_{1 / 2}$ (lower plot). There is no such combination of HS and LS fraction that can accurately reproduce the spectrum at the intermediate temperature, even when fractions are hugely exaggerated for best match, see upper plots in Fig. 2. This suggests that spectral kinetics of electronic transitions are affected by temperature of the electronic states between which these transitions occur. Band narrowing and spectral shifts are possible consequences of thermal excitation/relaxation of vibra- 
tional modes of molecules. We touch upon this point in the following section.

\subsection{Spectral snapshots away from equilibrium}

The main goal of our time-resolved study was to elicit the nature of photogenerated molecules in the metastable state populated within 300 fs following the FranckCondon excitation. Our earlier investigations [4] relied on picking out the sensitive wavelengths from the thermal crossover absorption spectra and relating those to the observed time-resolved optical transmission at those specific wavelengths. This methods yields high sensitivity and is free of dispersion artefacts [9] but lacks the discriminating power of broad bandwidth detection. In this report, we provide a broad bandwidth spectrum of photogenerated molecules by taking a certain number of precautions allowing reliable spectral disentanglement.
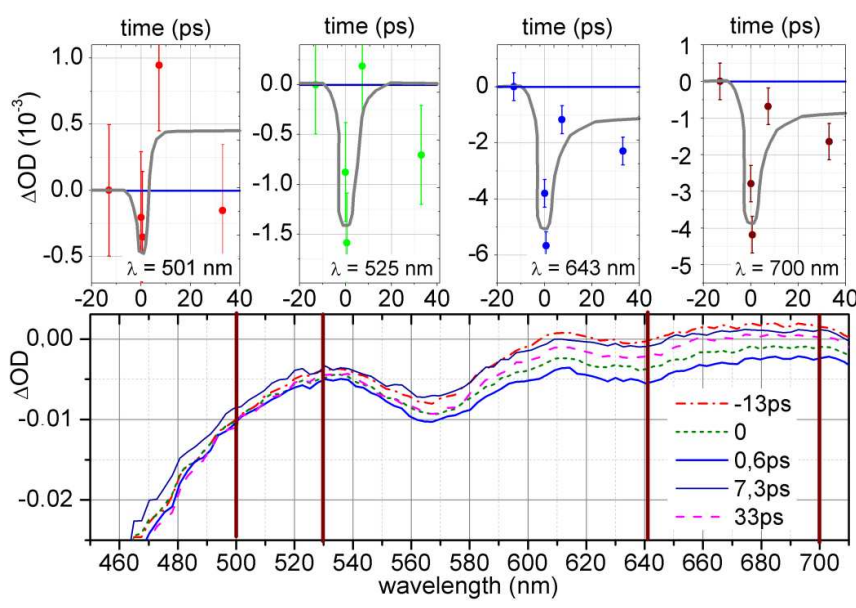

Fig. 3. Lower part: time resolved spectra at different time delays taken at $180 \mathrm{~K}$; upper part: spectral cuts at selected wavelengths (full circles) with errors bars from experiment, solid lines drawn to help guide the eye.

Figure 3 (lower part) shows time-resolved spectra taken at different time delays. The spectrum at a negative delay $(-13 \mathrm{ps})$, where probe precedes the pump, was taken twice to estimate the accuracy of the collected data. The entire spectrum is chirped, meaning there is a time lag between the leading edge at $710 \mathrm{~nm}$ and the trailing edge at the $480 \mathrm{~nm}$. This lag was measured in a separate experiment and equals $1.6 \mathrm{ps}$. This is consistent with the group velocity dispersion we measured, $10 \mathrm{fs} / \mathrm{THz}$. In keeping with this notation, time 0 is in the coincidence with the pump of the furthermost trailing wavelength of recorded white light. The longest delay recorded at 33 ps ensures that the chirp (1.6 ps) has negligible effect on the spectrum, as it was already demonstrated that the system does not evolve in the 2 ps- 1 ns time window [4] and so the spectral amplitudes do not have to be corrected for chirp. 33 ps delay also ensures that the system is probed long after LMCT relaxation, i.e. when all molecules having absorbed a photon and having gone this pathway evolve somewhere in the excited HS state potential.

Figure 3 (upper part) shows the sections of white light spectrum at wavelengths of which time course we established in our recent study using monochromatic detection [4]. For simplicity, these cuts were offset to zero for negative delays, while the origin of the offset will be discussed below. Within experimental accuracy, the comparison is satisfactory and proves that white light spectroscopy has sufficiently resolving power to monitor even minute changes of HS state concentration $(<1 \%$, from [4]). At longer wavelengths, the change of optical density due to increase of HS state fraction is greater than at shorter wavelengths, which explains why the blue side of the spectrum is less conclusive than its red side. The sign of OD change agrees with the change expected during thermal spin-crossover, see arrows in Fig. 1. Upon crossover from LS state to HS state the crystal becomes more transparent to light above $530 \mathrm{~nm}$ and more opaque below this wavelength. The cut at $525 \mathrm{~nm}$, which we earlier identified as the isosbestic point of the spectral kinetics, shows only variations around time zero and those must be related to the Franck-Condon transition and the resulting short-lived states.

The first and foremost advantage of time-resolved white light spectroscopy is a simultaneous recording of optical changes, here optical density, across a broad range of wavelengths. In a very general case we can observe band narrowing, overlapping, peak shifts, etc., which escape the monochromatic detection. The spectra in Fig. 3 are accumulated with the excitation rate of $250 \mathrm{~Hz}$, meaning that negative delays close to zero are equivalent to $4 \mathrm{~ms}$ after excitation. The spectrum at -13 ps clearly has a non-vanishing contribution despite $4 \mathrm{~ms}$ allowed for relaxation. However, from our earlier studies [10] we know that when the photoexcited crystal is kept at temperatures underneath thermal spin-crossover, the thermal conversion of LS molecules will result in additional and delayed yield of HS species. The relaxation of these switched molecules obeys heat exchange with the sample environment (cryogenic flow) to recover thermal equilibrium between sample and the environment. Because the related macroscopic time scale is longer than the excitation period, the crystal remains at a temperature slightly above that of the environment, for as long as the laser is on the sample. The residual spectrum at negative delays is a signature of thermally generated fraction of high-spin molecules.

Our major goal in this study was to obtain a snapshot of a pure photogenerated HS state, and see whether it differs from the HS state of the $100 \%$ HS crystal converted thermally. If so, we would like to understand how the differences come about and how they can be accounted for.

Figure 4 (lower part) plots the optical density spectrum of the thermally populated HS state (dotted line) superimposed on the spectrum taken 33 ps following excitation (solid line). The former had to be scaled down by 


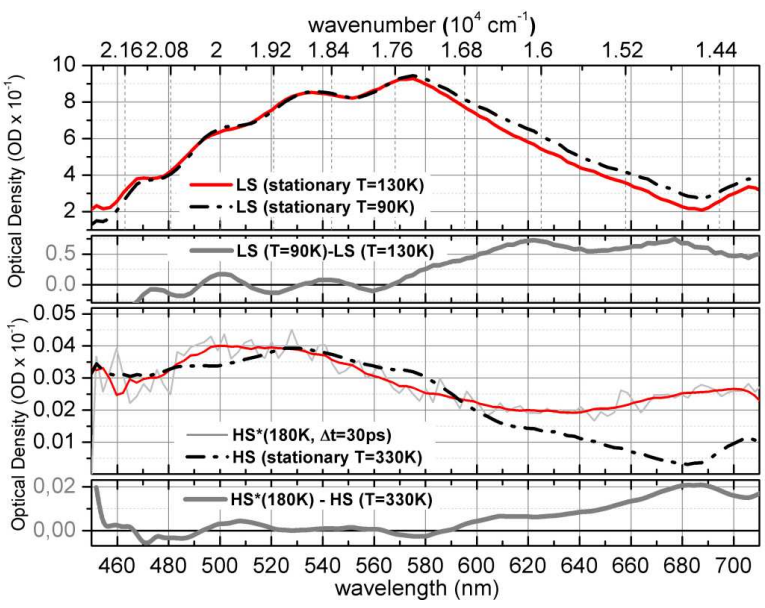

Fig. 4. Upper part: superposition of LS spectra taken at $90 \mathrm{~K}$ (black dashed) and $130 \mathrm{~K}$ (red solid), the residual difference signal is shown underneath; lower part: time resolved spectrum taken at $180 \mathrm{~K}$ and $30 \mathrm{ps}$ after laser excitation (red solid) superimposed on HS spectrum at $330 \mathrm{~K}$ (black dashed), the residual difference signal is shown underneath.

a factor of 171 so as to level the values of both spectra at the isosbestic point, which arguably is the most appropriate point in the spectrum for normalizing the molecular concentrations, rather than spectral intensities. This factor provides valuable information on the number of HS fraction induced by the laser, and equals $0.6 \%(1 / 171)$ in our study. The residual signal resulting from the difference between photoinduced and thermally generated HS is provided under the spectra. Even though both spectra have very similar shapes their spectral weight is not exactly the same throughout the VIS range.

To better understand the origin of this deviation we compare two spectra of a crystal in $100 \%$ LS state, but at different temperatures, Fig. 4 (upper part). We know from magnetic measurements that the fraction of LS molecules at $90 \mathrm{~K}$ and $130 \mathrm{~K}$ are practically the same and almost 1 . Yet, the spectra at these temperatures are slightly different, and that difference cannot be trivially attributed to intensity fluctuations. The two were superimposed in the manner discussed above, which implies that the residual difference cannot be due to the molecular concentration fluctuation either. The residual signal between 90 and $130 \mathrm{~K}$ of LS state spectra shows almost exactly the same deviation as that of the photoinduced HS state at $180 \mathrm{~K}$ from thermally converted HS state at $330 \mathrm{~K}$. We believe that these spectral deviations are due to thermal population of vibrational levels of the electronic potentials of the HS and LS states. To quantify these effects dedicated studies using far-IR techniques would be necessary. However, we can conjecture that the HS state generated at $180 \mathrm{~K}$ by a laser pulse is vibrationally cooled within $30 \mathrm{ps}$ where it settles in its electronic potential. The "cold" HS molecules evolve in this potential with less thermal energy than when the crystal is $100 \% \mathrm{HS}$ at temperatures above $330 \mathrm{~K}$. In view of the growing interest in the control over solids excited to thermodynamically inaccessible physical states, efforts promoting ultrafast IR spectroscopy $[11,12]$ or picosecond Raman [13] will be timely.

\section{Conclusions}

We have reported on the observation of a transient state in a molecular crystal by using time resolved optical pump and supercontinuum probe technique. With the spectral snapshots taken prior to and 30 ps after femtosecond laser excitation we could identify the transient as being that of a "cold" HS state photogenerated at $180 \mathrm{~K}$. We also observe that "cold" HS and "cold" LS deviate spectrally from "hot" HS and "hot" LS in almost exactly the same way, as they each bear a vibronic contribution showing in the VIS absorption.

\section{Acknowledgments}

This work was supported by the ANR (09BLAN-0212) and Europe (FEDER).

\section{References}

[1] P. Gutlich, H.A. Goodwin, Spin Crossover in Transition Metal Compounds I - III, Springer, 2004.

[2] P. Gutlich, A. Hauser, Angew. Chem. Int. Ed. Engl. 33, 2024 (1994).

[3] N. Moisan, M. Servol, M. Lorenc, A. Tissot, M.L. Boillot, H. Cailleau, S. Koshihara, E. Collet, C.R. Chimie 11, 1235 (2008).

[4] M. Lorenc, J. Hébert, N. Moisan, E. Trzop, M. Servol, M. Buron, H. Cailleau, M.L. Boillot, E. Ponte, M. Wulff, S. Koshihara, E. Collet, Phys. Rev. Lett. 103, 028301 (2009).

[5] E. Collet, M.L. Boillot, J. Hebert, N. Moisan, M. Servol, M. Lorenc, L. Toupet, M. Buron-le Cointe, A. Tissot, J. Sainton, Acta Crystallogr. B 65, 474 (2009).

[6] R. Naskręcki, Femtosecond Transient Absorption Spectroscopy. Photophysical Study of the Excited States of Molecules and Short-Living Individuals, Wydawnictwo Naukowe UAM, Poznań 2000.

[7] A.J. Simaan, M.L. Boillot, R. Carrasco, J. Cano, J.-J. Girerd, T.A. Mattioli, J. Ensling, H. Spiering, P. Gütlich, Chem. Eur. J. 11, 1779 (2005).

[8] A. Dei, D. Gatteschi, L. Pardi, Inorg. Chem. 32, 1389 (1993).

[9] M. Ziółek, M. Lorenc, R. Naskręcki, Appl. Phys. B 72, 843 (2001).

[10] H. Cailleau, M. Lorenc, L. Guérin, M. Servol, E. Collet, M. Buron-Le Cointe, Acta Crystallogr. A 66, 189 (2010).

[11] T. Elsaesser, W. Kaiser, Ann. Rev. Phys. Chem. 42 , 83 (1991).

[12] Y. Zhang, J. Kubicki, M.S. Platz, J. Am. Chem. Soc. 131, 13602 (2009).

[13] J.D. Leonard, J. Phys. Chem. A 105, 1724 (2001). 